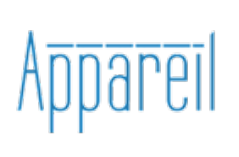

Appareil

2| 2008

Autour de Simondon

\title{
La zone obscure des nanotechnologies
}

\section{Sacha Loeve}

\section{(2) OpenEdition}

\section{Journals}

Édition électronique

URL : http://journals.openedition.org/appareil/635

DOI : 10.4000/appareil.635

ISSN : 2101-0714

\section{Éditeur}

MSH Paris Nord

\section{Référence électronique}

Sacha Loeve, «La zone obscure des nanotechnologies », Appareil [En ligne], 2 | 2008, mis en ligne le 02 octobre 2008, consulté le 30 juillet 2020. URL : http://journals.openedition.org/appareil/635 ; DOI : https://doi.org/10.4000/appareil.635

Ce document a été généré automatiquement le 30 juillet 2020.

\section{(c) (i) (2) $\Theta$}

Appareil est mis à disposition selon les termes de la Licence Creative Commons Attribution - Pas d'Utilisation Commerciale - Pas de Modification 4.0 International. 


\title{
La zone obscure des nanotechnologies
}

\author{
Sacha Loeve
}

\section{NOTE DE L'AUTEUR}

\section{Remerciements :}

Jean-Pierre Sauvage, Jean-paul Collin, Julien Frey, Pierre Moebian, Benoît Champin, Patrice Staub et toute l'équipe du Laboratoire de Chimie Organo-Minérale (LCOM), Strasbourg.

Gérald Dujardin, Damien Riedel, Franco Chiaravallotti et Andrew Mayne au Laboratoire de PhotoPhysique Moléculaire (LPPM), Orsay.

Jean-Claude Moutet, Guy Royal, Christophe Bucher et Damien Jouvenot, au Laboratoire d'Electrochimie Organique et de Photochimie Redox (LEOPR), Grenoble.

Christian Joachim, Xavier Bouju, Gwénaël Rapenne, Guillaume Vives et Sébastien Gauthier au Centre d'Élaboration des Matériaux et des Systèmes (CEMES), Toulouse.

\section{Introduction}

Le schéma hylémorphique correspond à la connaissance d'un homme qui reste à l'extérieur de l'atelier et ne considère que ce qui y rentre et

ce qui en sort ; pour connaître la véritable relation hylémorphique, il ne suffit pas même de

pénétrer dans l'atelier et de travailler avec l'artisan : il faudrait pénétrer dans le moule luimême pour suivre l'opération de prise de forme aux différents échelons de grandeur de la réalité

physique ${ }^{1}$.

Gilbert Simondon 
1 Les nanotechnologies ${ }^{2}$, censées inaugurer une nouvelle révolution industrielle, souffrent du même manque. Au lieu de connaissances sur le fonctionnement et la genèse des nano-objets, c'est le schéma hylémorphique critiqué par Simondon que l'on retrouve $^{3}$. En effet, on a tendance à se représenter les nanotechnologies de la manière suivante : ce qui entre au laboratoire (en l'occurrence des atomes et des molécules) est considéré comme de la matière disponible et docile ; ce qui en sort, on considère que ce sont des objets utiles, identifiables par leur forme et par leur finalité, en référence à des objets bien connus (levier, véhicule, moteur, etc.). Or l'appréhension de ces objets à l'aide des couples d'opposition classiques forme/matière et fin/moyen nous laisse, pour paraphraser Simondon, face à une zone obscure. Ce qui est occulté, c'est précisément l'opération d'individuation, c'est-à-dire dans le cas d'une machine les processus de sa genèse et de son fonctionnement. Or avec les nanotechnologies, il s'agit précisément d'intervenir dans l'opération d'individuation elle-même, ou de susciter d'autres individuations. Dans la mesure où les pratiques instrumentées des nanotechnologies positionnent le chercheur plus près des molécules qu'il manipule, elles exaucent d'une certaine manière le vœu émis par Simondon, celui de suivre l'opération d'individuation de l'intérieur du moule. Le problème, pour les chercheurs, serait bien plutôt d'en sortir. En effet les deux mondes, le «nanomonde" et le nôtre, communiquent très mal. Pourtant ce n'est pas la communication qui manque aux nanotechnologies. Comment expliquer cette situation? Les nanotechnologies ont beau être intensément médiatisées, les pratiques nanotechnologiques ne sont pas symbolisées, et c'est sur leur occultation que sont fondés les grands discours et la communication des nanotechnologies. Tout se passe comme si l'artisan dont parle Simondon, muni d'instruments lui permettant d'entrer dans le moule pour suivre l'opération d'individuation du dedans, y était maintenu confiné. Comme s'il ne lui était permis d'en sortir qu'après avoir effacé toute trace de l'argile sale sur la combinaison futuriste qu'on lui a généreusement financée.

2 On présentera d'abord trois lignes de force de ces grands discours : la convertibilité mutuelle du naturel et de l'artificiel, l'exacerbation du pôle anthropologique, l'instrumentalisation de la science-fiction comme stratégie de cryptotechnicité. Puis on décrira quelques pratiques dans le domaine des machines moléculaires ${ }^{4}$. On montrera ce que signifie une individuation technologique à l'échelle moléculaire. Enfin on s'interrogera en conclusion sur le rapport de ces pratiques et de ces discours.

\section{Petites machines et grands discours}

\section{La convertibilité mutuelle du naturel et de l'artificiel}

3 Les propos rapportés ici, extraits de brochures éducatives, sont deux exemples parmi d'autres des discours « rassurants » que l'on trouve à foison.

Une leçon de la nature - Les atomes sont les briques élémentaires de construction de toute la matière de notre monde naturel. (...) Les atomes se lient ensemble pour former des structures moléculaires. (...) La molécule est la plus petite partie, le composant ultime qui se trouve être doté d'un ensemble de propriétés physiques et chimiques uniques. Les molécules composent aussi bien l'air que nous respirons, l'eau que nous buvons, et même les cellules vivantes de nos corps - ce sont les nanomachines de la nature. Le but des nanotechnologies est d'être capable de manipuler chaque atome ou chaque molécule un par un, de leur faire former des 
motifs afin d'obtenir la structure désirée - tout comme un arrangement de briques de LEGO ${ }^{\mathrm{TM} 5}$.

La nanotechnologie dans la nature - Les nanotechnologues aiment la nature. En quatre milliards d'années, celle-ci a en effet trouvé des solutions parfois étonnantes à ses problèmes. Un exemple typique réside dans la façon dont la vie structure sa matière jusque dans les détails, jusqu'au niveau des atomes. C'est également ce que recherchent les nanotechnologues. [...] Personne ne doit avoir peur de la complexité de la nanotechnologie. La pomme est un ensemble complexe - cellules, ribosomes, ADN - mais elle reste un fruit fort apprécié. À l'instar des bonnes nanotechnologies, la pomme se manipule aisément ${ }^{6}$.

4 Rien n'est à proprement parler faux dans de telles affirmations. Tout se passe au contraire comme s'il s'agissait d'idées si évidentes qu'elles ne valent pas même la peine d'être questionnées ou justifiées rationnellement. Elles relèvent moins d'un registre épistémologique que d'un registre de justification: reconnues "naturelles", les nanotechnologies peuvent être considérées d'emblée comme acceptables et inévitables. La question elle-même n'a plus de sens si elles existent déjà dans la nature. Par une sorte de "sophisme naturaliste ${ }^{7}$ ", on passe sans plus de difficultés de l'être au devoirêtre en invoquant le temps immémorial de l'Évolution. Comment ces discours procèdent-ils ? Ils affirment d'abord que la nature fonctionne à l'échelle nanométrique comme une sorte de nano-ingénieur; si la nature a développé les nanotechnologies depuis au moins l'apparition de la vie, il est par conséquent possible de reproduire ces mécanismes ou de les améliorer; imiter la nature, en tentant de répliquer ses mécanismes, ne revient qu'à s'inscrire dans son mouvement même. Une fois la nature et la vie préalablement redéfinies en termes nanotechnologiques, le processus d'innovation technologique est lui-même naturalisé.

5 En quoi cette naturalisation de l'artifice est-elle différente de celle qu'opère Simondon lorsqu'il dit l'objet technique porteur d'« une charge de nature »? En effet, lorsqu'il évolue en se concrétisant, la récurrence de causalité dans la relation de l'objet au milieu associé le rapproche asymptotiquement du mode d'existence des êtres vivants, explique Simondon. C'est ce qui fait que la technologie peut comme la biologie pratiquer une étude inductive plutôt que déductive (partant de principes a priori et les appliquant). Nous pensons que ce thème est chez lui indissociable de la reconnaissance de la singularité du régime d'individuation technique - il faut reconnaître des modes d'existence spécifiquement techniques, fut-ce par analogie avec la biologie. Qui dit « analogie» ne dit pas relation d'identité, mais identité de relation. Autrement dit, l'analogie avec l'évolution du vivant, lorsqu'elle privilégie les relations entre fonctions plutôt que les métaphores basées sur des assimilations ${ }^{8}$, permet de penser un régime d'individuation spécifiquement technologique9. L'évolutionnisme des nanotechnologies apparaît beaucoup plus pauvre. Il ne cherche pas à dégager les conditions d'intelligibilité de l'évolution de ces objets, leur signification technologique, mais simplement à justifier leur avènement par le flot de l'Évolution. L'idéologie diffère de celle des vieux évolutionnismes. L'ancien télos du progrès technique, qui faisait soupçonner l'évolutionnisme technique d'être la justification d'une certaine vision de l'émancipation culturelle, ne se rencontre plus. À la place de l'évolution technique, on trouve un processus décrit comme naturel et entropique. À la place de la nature, on trouve... la technologie, avec des affirmations comme «la biologie, ce sont les nanotechnologies qui marchent» ou «la preuve que les nanotechnologies sont possibles, c'est que nous sommes là ». Le diagnostic peut donc paraître paradoxal : les 
concepts de nature et d'artifice ne fonctionnent plus, tout en étant, plus que jamais, surinvestis de mythe.

\section{L'exacerbation du pôle anthropologique des nanotechnologies}

6 Dans les grands discours, une figure anthropologique est mise en avant, celle de l'«humain augmenté » qui modifie son processus d'évolution par l'intégration des techniques à même son individualité somatique et psychique ${ }^{10}$. Faut-il y voir le signe d'une ouverture anthropologique inaugurant un nouveau type de lien social intégrant les non humains? On peut en douter. Au contraire, tout se passe comme si cette figure exprimait le vœu de l'humain de revenir à son essence, de rapatrier ce qu'il avait extériorisé et objectivé ${ }^{11}$, outils et objets techniques, pour coïncider avec lui-même dans la transparence. Les produits NBIC, censés être totalement personnalisables et transparents à leurs utilisateurs, ne sont destinés qu'à être l'expression parfaite du moi de l'homme augmenté, individu insulaire, coupé du monde et des autres dont on veut faire le consommateur rêvé de demain. C'est bien ce "revenir à soi » que semble traduire cette volonté d'ingestion des artefacts; cela revient à vouloir résorber la réalité humaine et ses artefacts ${ }^{12}$ dans des individus sujets-objets de consommation.

7 Loin d'exprimer une réconciliation de l'homme et d'une "techno-nature", l'homme augmenté est un épouvantail qui cache une béance: d'un côté le sujet est placé au centre de ses technologies, en situation de maittre, pour affirmer le contrôle psychique et somatique qu'il possède sur leur mise en œuvre, comme si celles-ci n'étaient que des outils au service de l'homo faber; l'objet technique est réduit à l'outil ; le monde est réduit à une réserve de matières premières. On parle ainsi d'assemblage des «briques élémentaires» de la nature, mises en forme avec la précision du «scalpel nanométrique ». L'individualité, l'eccéité de l'objet ne lui est accordée qu'au prix d'une projection de l'intention fabricatrice et de l'intention de consommation, sans que l'on ne thématise jamais l'objet comme tel. On a donc un sujet apparemment «technicisé ", non seulement porteur d'outils, mais objet de l'opération outillée dont il est le sujet. D'un autre côté l'humain sujet-objet est sans monde d'objets extérieurs (et peut être sans sujets extérieurs). Ce n'est pas un hasard si, dans le rapport NBIC, les qualités humaines sont réduites à une quantité de vitalité, les savoir-vivre à de la compétitivité et les droits sociaux aux performances individuelles et à celles du corps social. Le « transhumain » de « l'humanité 2.0 » prophétisée par Ray Kurtweil, censé transcender ses limites corporelles, est en fait complètement absorbé dans la nature - ou complètement absorbé dans la société, le tout-artifice étant ici équivalent au toutnature ; il ne vit plus que sur un seul plan. Lorsque les objets du monde ont disparu, ce qui est de l'ordre du culturel, du symbolique et du récit disparaît dans l'insignifiance et la futilité ; bios est réduit à zoè ; la civilisation est comme engloutie dans la nature. Comment peut-on dans ce cas parler d'" augmentation de nos capacités »?

Plus qu'une critique morale de cette figure, c'est la question du statut des objets qui nous retient ici. Entre inscription de la volonté humaine toute puissante dans l'intimité de la matière et négation de l'humain par des processus autonomes ${ }^{13}$, les nano-objets semblent ne pas trouver leur place. L'humain augmenté n'a jamais affaire à de la technique, mais à des interfaces sans épaisseur agissant comme des narcotiques. Il ne se soucie plus ni de la technique, ni du monde. Il s'agit donc d'une crise symbolique de notre rapport aux objets techniques. Non qu'elle soit causée par les nanotechnologies : 
mais en posant plus intensément la question du rôle des objets techniques dans la constitution du monde humain, celles-ci jouent le rôle d'un révélateur. Ce qui fait la pauvreté des discours transhumains, c'est qu'ils prétendent parler de l'humain et de son rapport aux technologies comme si la relation entre les deux pouvait être directe ${ }^{14}$, sans passer par l'inclusion des humains dans un système technique qui les relie via un maillage des milieux culturels/naturels. Dit à la manière de Simondon, ce sont des discours anthropologiques et non technologiques. Ils n'assument pas d'autre point de vue que celui centré sur l'homo ou sa négation. Dit à la manière d'Hannah Arendt, ce sont des discours sur la nature humaine et non sur la condition humaine - c'est-à-dire des discours sur l'essence métaphysique de l'homme et non sur la manière dont il conditionne son existence en participant à l'édification d'un monde commun et partagé.

Les discours transhumanistes sont donc des discours normatifs. Mais leurs assomptions sont liées à une manière d'appréhender les objets. C'est sur ce plan que nous voudrions les placer en insistant sur le "souci du monde" auquel devraient nous engager ces technologies, à l'opposé de la figure du pur individu-consommateur des NBIC.

\section{La science-fiction comme stratégie de cryptotechnicité}

Les procédés de mise en récit des nanotechnologies les plus utilisés par ces discours sont ceux de la science-fiction. Il n'est pas question ici de s'étonner de ce qu'un genre littéraire puise son imaginaire dans un champ technoscientifique contemporain comme il a pu le faire avec l'intelligence artificielle et la conquête spatiale. C'est là sa définition même. Que la fiction nourrisse la culture des chercheurs, qu'ils l'utilisent pour oser aller plus loin $^{15}$, cela n'a rien de scandaleux non plus. Or les discours futuristes sont moins tenus par les chercheurs que par les politiques de la recherche et des entrepreneurs : les stratégies narratives du récit de science-fiction sont délibérément instrumentalisées par la rhétorique des discours officiels, pour des fins de communication avec le public, pour catalyser la recherche, stimuler l'investissement et créer un horizon d'attente visionnaire. «Qu'en est-il des développements en ingénierie fondés sur la science actuelle mais qui s'appuieront sur des capacités à venir ? N'est-ce pas l'ouverture d'un vaste champ d'investigation ${ }^{16}$ » Le souhait d'Éric K. Drexler a été largement exaucé. Dorénavant, les chercheurs doivent concevoir des systèmes basés sur l'anticipation des possibilités techniques à venir, étant donné un cadre scientifique relativement stable ${ }^{17}$. Ils sont donc poussés en somme à déguiser constamment les objets produits en fonction de leur ressemblance avec un objet futur. Pour qu'une recherche " nano » non directement applicative ${ }^{18}$ soit valorisée, il faut que sa technicité actuelle puisse s'effacer et passer pour une conséquence de la technologie du futur. Il ne s'agit de rien de moins que d'exploiter et de capitaliser les visions du futur pour leur faire produire «à rebours » les technologies du présent. Cela marche-t-il réellement? Peu importe! En attendant, il suffit de déguiser les productions du présent en retombées du futur.

11 Que les objets techniques aient besoin de se déguiser pour entrer en société, qu'une société se définisse par les techniques qu'elle accepte ou non d'accueillir dans son collectif ainsi que par le jeu de dissimulation et d'ostentation qu'elle leur impose, c'est ce qu'avance Simondon dans Psycho-sociologie de la technicité :

Quels sont les critères de l'ostracisme qui frappe les objets techniques? Le plus constant est l'obligation de porter un voile ou un déguisement pour pénétrer dans 
la citadelle de la culture; ce voile ne fait point illusion, mais il maintient la séparation entre le sacré et le profane [...]. Généralement, les objets phanérotechniques [objets qui exhibent leurs parties fonctionnelles] sont considérés comme utilitaires (par exemple, les moto-pompes, les groupes électrogènes, les motoculteurs, certains tracteurs, ont un moteur apparent), tandis que les objets cryptotechniques sont susceptibles d'être introduits dans la citadelle de la culture ${ }^{19}$.

C'est ce que Simondon nomme la cryptotechnicité: le fonctionnement des objets techniques doit être caché pour ne transparaître que dans une pseudo-technicité rappelant des scènes familières. C'est à cette condition que les nouveaux objets techniques peuvent avoir droit de cité dans la culture ${ }^{20}$. Simondon évoque par exemple la simulation du feu du foyer, sur les nouveaux radiateurs électriques dans les années 1960. Certes, les objets que décrit Simondon doivent revêtir des archétypes du passé, alors que les nôtres, telle la machine moléculaire devant ressembler à un nanorobot (figure 1), doivent véhiculer des archétypes du futur. Mais cela est-il vraiment le futur? Qu'est-ce que la science-fiction sinon la projection dans l'avenir d'un passé qui n'a jamais eu lieu? Un futur antérieur puisant dans un répertoire de formes bien connues? Ainsi, si les machines moléculaires doivent ressembler au nano-robot que l'on rencontre sur Internet, c'est encore pour projeter une scène familière. Bien souvent, la figure du nano-robot est la projection déplacée dans d'autres contextes du schéma corporel de l'homme au travail ${ }^{21}$.

Figure 1 : d'hypothétiques nanorobots au travail

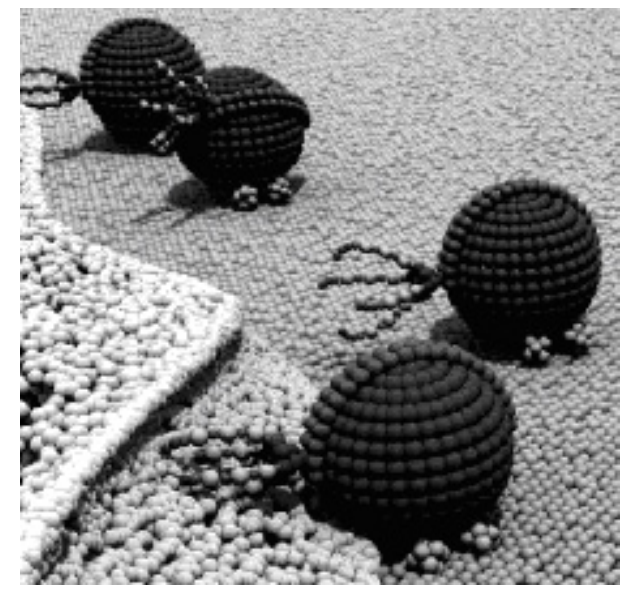

Source : CEMES

La figure 1 est une capture d'image prise sur un film d'animation. Il nous a été communiqué par un chimiste qui synthétise des machines moléculaires. Il s'agissait pour lui d'essayer de se figurer à quoi pourraient ressembler des nanorobots en termes chimiques. Il en concluait qu'il était bien incapable de synthétiser une telle molécule et que de plus, il ne voyait absolument pas comment celle-ci pouvait fonctionner. Or son travail est de synthétiser des molécules qui ont un fonctionnement. Mais pour les discours officiels, tout cela importe peu! En surinvestissant les nano-objets de caractéristiques futuristes, on parie que cela produira des effets. Car c'est bien un pari : l'anticipation des possibles futurs en fera passer quelques-uns à l'existence. Les promoteurs des nanotechnologies ne se soucient plus du degré de plausibilité des anticipations lorsqu'elles sont émises. Ils attendent d'elles, à force de les marteler, 
qu'elles deviennent des prophéties auto-réalisatrices. Mais ce carnaval des objets finit par parodier les discours ${ }^{22}$.

Simondon nous apprend que la manière dont on négocie avec la technicité ses stratégies de dissimulation et d'ostentation - ce qu'il appelle ailleurs son aura - définit une société. La manière dont la promotion des nanotechnologies s'organise en excluant d'avance l'idée que le «public " puisse s'intéresser à autre chose qu'à des applications inouïes, infiniment utiles et incroyablement rentables et non à leur technicité intrinsèque court-circuite d'avance le collectif en le fondant sur un jeu de dupes et sur une déréalisation du présent technologique. C'est pourquoi seule l'analyse du mode d'existence des nano-objets, y compris de leur devenir à travers des images intra- et extra-scientifiques, est susceptible d'introduire du déphasage dans ce cercle spéculatif. En rebranchant les discours hype sur les objets qui les suscitent, nous voudrions résister à une autre tentation : celle de démystifier en traçant une ligne de démarcation nette entre vérité scientifique et fiction. Les problèmes de symbolisation de ces objets ne viennent pas que de préjugés humains ou de causes sociales, mais également - nous le montrerons - de ces objets eux-mêmes.

\section{Les machines moléculaires: une individuation technologique à l'échelle moléculaire}

\section{Individuer la molécule}

Une des approches pour la synthèse de machines moléculaires est la chimie de coordination $^{23}$. Au Laboratoire de Chimie Organo-Minérale (LCOM) à Strasbourg, l'équipe de Jean-Pierre Sauvage s'est distinguée dans la réalisation de machines moléculaires comme les caténanes bistables (figure 2). Ce sont des bistables rédox, c'est-à-dire activés par oxydation et réduction (on plonge des électrodes dans une solution contenant des milliards de ces molécules; mais d'autres machines moléculaires sont activées à la lumière ou au pH). Selon que l'ion métallique, maintenu au centre de la molécule par des liaison métal-ligand, perde ou gagne un électron, il est possible de contrôler (collectivement, et non molécule par molécule) le mouvement en jouant sur ce qu'on appelle le nombre de coordination de l'ion; selon son état, réduit ou oxydé, il va " préférer » se lier à une autre ligand sur la molécule en fonction de la géométrie de ses électrons de valence (figure 3). Une partie de la molécule va alors basculer pour adopter une autre conformation. 
Figure 2 : bistable moléculaire constitué de deux caténanes entrelacées

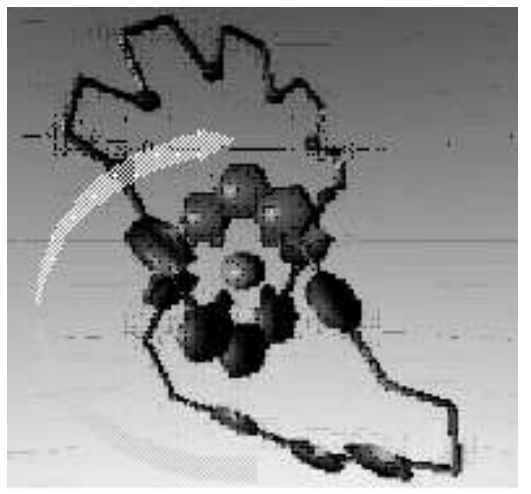

Source : Jean-Pierre Sauvage, LCOM.

Figure 3 : ligands

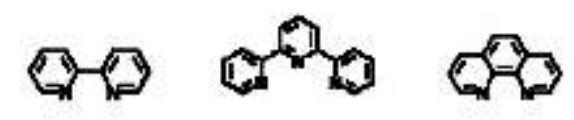

De gauche à droite : Bipyridine, dite "bipy" (2,2'-bipyridine) ${ }^{24} \mathrm{C10H8N2}$, Terpyridine dite "terpy" (2,2' ;6'2"terpyridine) C15H11N3 phénanthroline dite "phen" (1,10-phénanthroline3) C12H8N2

16 Faire de telles molécules, disent les chimistes, relève de " défis de synthèse », tant elles sont exotiques et d'une haute improbabilité. L'existence de chacune d'elles est ainsi considérée comme une sorte de miracle. Aucune application à court terme n'est envisagée pour ces machines, dont existent de nombreuses variétés (voir par exemple le shuttle moléculaire de la figure 6 et le complexe de cuivre de la figure 7). Les finalités applicatives ne sont pas prises en compte dans la conception de ces objets (du moins par les chercheurs qui les font). Ajoutons que cela ne veut pas dire qu'ils ne donneront jamais lieu à des applications, mais simplement qu'il ne s'agit pas de recherche applicative, même s'il s'agit de faire des machines.

17 Le principe et les étapes du fonctionnement (figure 4) sont faciles à comprendre : le $\mathrm{Cu}(\mathrm{I})$ est tétracoordinné, c'est-à-dire qu'il se lie de préférence à quatre atomes d'azote (N). L'ion $\mathrm{Cu}(\mathrm{II})$ est pentacoordinné, il « préfère » se lier à cinq atomes d'azote. Lors de l'oxydation de la solution, l'ion cède des électrons, et le complexe devient instable ; l'anneau se libère, jusqu'à ce que l'ion Cu(II) aille se lier à cinq atomes d'azote dans une géométrie plus stable. En réduisant, l'ion métal regagne un électron, $\mathrm{Cu}(\mathrm{II})$ redevient $\mathrm{Cu}(\mathrm{I})$. Celui-ci va "vouloir" retrouver ses quatre atomes d'azote au lieu des cinq auxquels il était lié. Il repasse d'un état pentacoordinné à un état tétracoordinné. L'anneau refait donc une rotation de $180^{\circ}$. L'opération est considérée comme une fonction; elle est réversible, mais le sens de rotation n'est pas dirigé ; le contrôle est collectif et stochastique, le mouvement de la machine est "causé » autant par la modification des énergies de liaison que par le mouvement brownien; la machine ne peut fournir aucun travail - ce n'est pas un moteur! la molécule passe d'un état d'équilibre à un autre sans maintenir un gradient d'énergie qui pourrait être libéré pour une production de travail. 
Figure 4 : Étapes de fonctionnement du bistable moléculaire

rotation of a ring within another ring (no directionality):

use of the Cu(II)/Cu(I) couple (Lvorell et al., 1994)

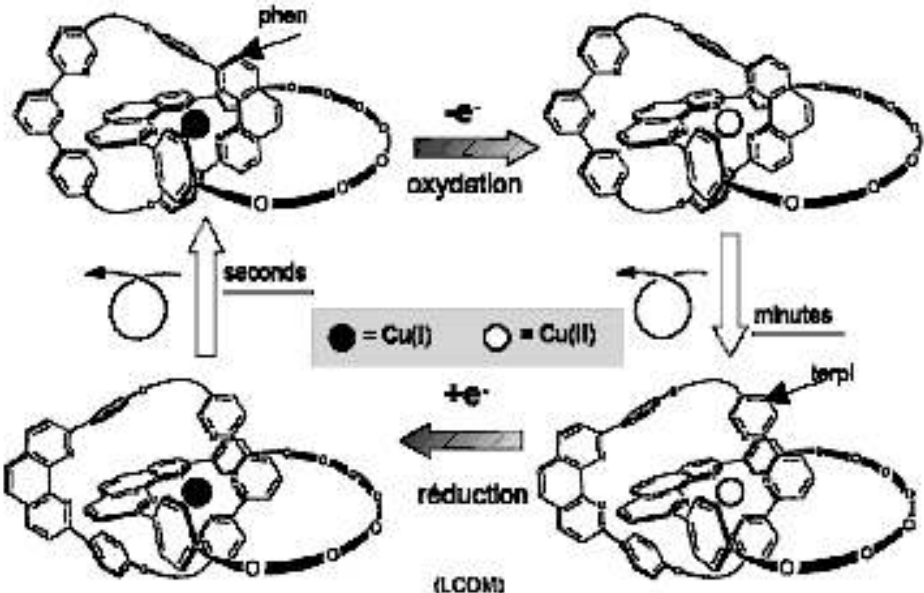

Source : présentation de Jean-Pierre Sauvage, LCOM.

On a donc des molécules bistables, des caténanes capables d'oscillation contrôlée. Posons la question suivante, pour bien comprendre ce que signifie «machine moléculaire » : à quelles conditions a-t-on une machine ? Avoir une molécule bistable, qui peut être, pour une population donnée dans une solution, statistiquement dans un état $A$ ou dans un état $B$, cela ne suffit pas. C'est ce que les chimistes appellent avoir une « assemblée » de molécules considérées comme des «systèmes dynamiques ». Mais un système dynamique n'est pas encore une machine. On sait que les molécules effectuent un mouvement, mais tant que l'on s'en tient à un diagramme ou à un décalque prélevant certains des traits de la population d'une solution, on n'a pas encore une machine.

Figure 5 : shuttle moléculaire

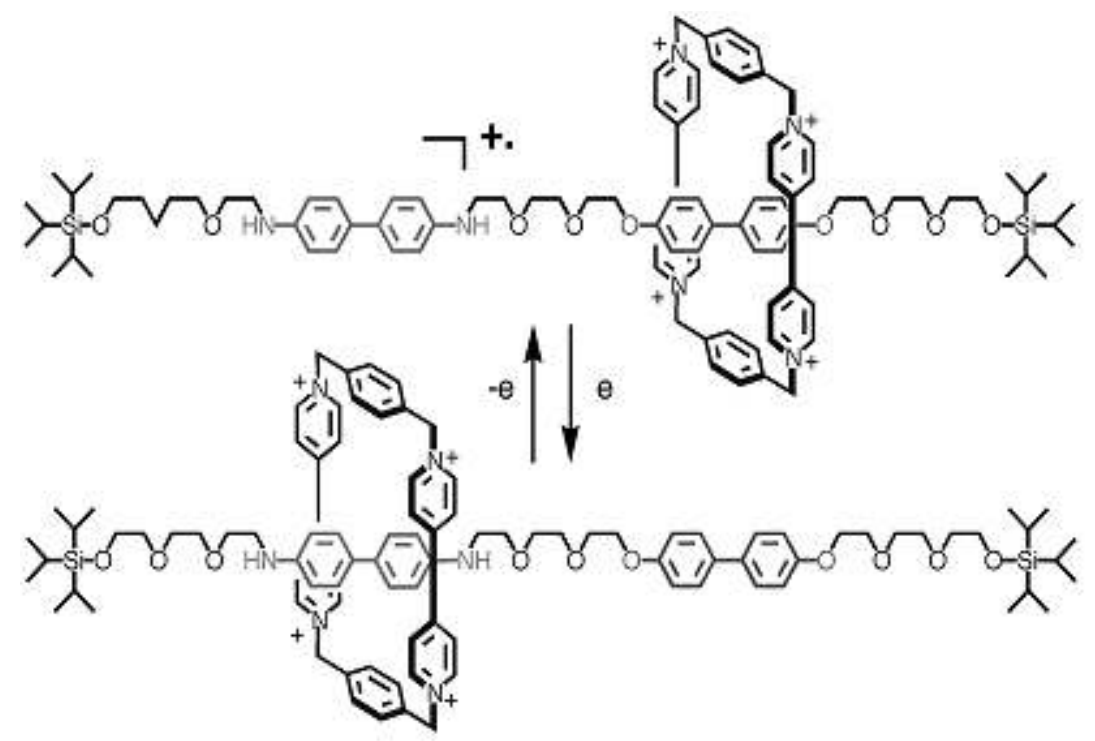

Bissel, Córdova, Kaifer, Stoddart, 1994. Présentation de Jean-Pierre Sauvage, LCOM. 
Figure 6 : caractériser le changement de conformation par l'interprétation des courbes d'électrochimie
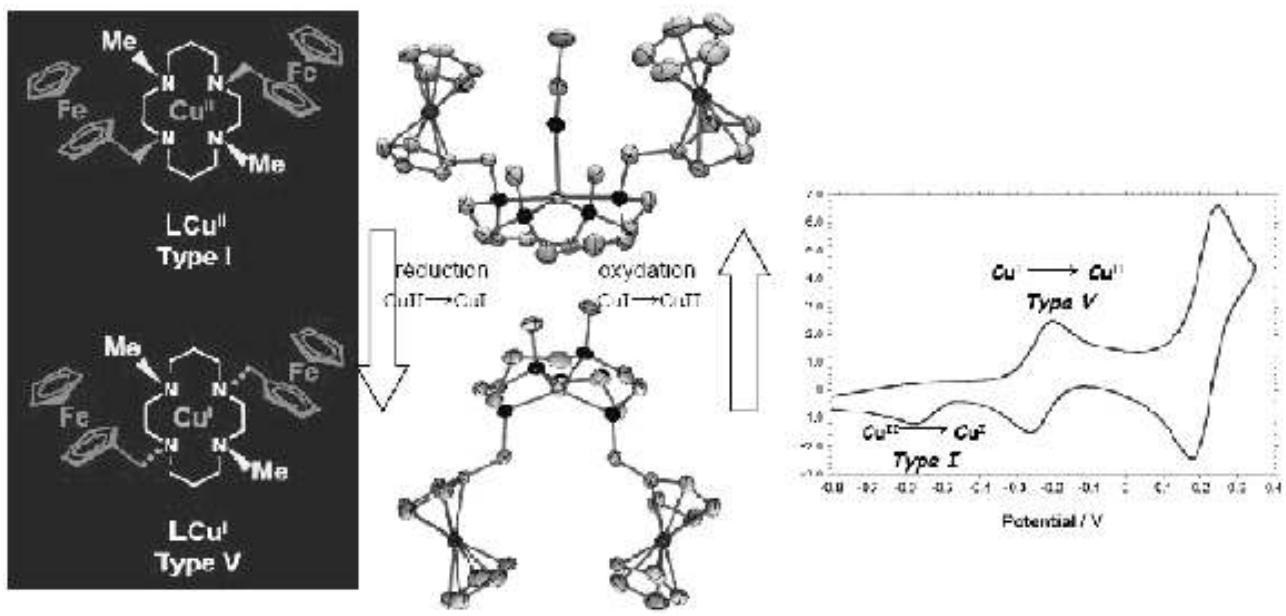

Montage réalisé à partir d'une présentation de Guy Royal, LEOPR.

SOURCE: GUY ROYAL, CHRISTOPHE BUCHER, JEAN-CLAUDE MOUTET ET ÉRIC SAINT-AMAN, « REDOXTRIggERED MOLECULAR MOVEMENT IN A MULTICOMPONENT METAL COMPLEX IN SOLUTION AND IN THE SOLID STATE ", 2004.

La chimie des machines moléculaires a pour objet la synthèse et la caractérisation de molécules dotées de fonctions; «fonction » ne signifie pas d'abord «utilité » (moyen pour une fin), mais schème fonctionnel; rotation et translation, sont les schèmes plus simples. Cette attention aux fonctions implique quelque chose de plus que la représentation des populations de molécules dans des formules chimiques: une opération de va-et-vient entre l'état collectif des molécules donné par la mesure (soit pour un bistable, le fait d'avoir un pourcentage de molécules dans un état A et un autre dans un état $\mathrm{B}$ à un moment $t$ ) et le devenir des molécules prises individuellement. On va parler de machine dès lors qu'entre l'état $A$ et l'état $B$, on va se demander comment se comporte la molécule. On passe de la représentation des propriétés statistiques à la schématisation du fonctionnement. La question du fonctionnement est loin d'être triviale : la molécule va-t-elle un peu vers $A$, puis un peu vers $B$, pour ensuite se fixer sur A ? Oscille-t-elle constamment de A à B et ne se stabilise-t-elle que dans une sorte d'état limite? Que se passe-t-il entre deux mesures? Entre les stations? Comment être certains que ce sont les mêmes molécules qui sont mesurées en $\mathrm{A}$ à un moment $t 1$ et en $\mathrm{B}$ à un moment $t 2$ ? À quels changements de conformation des molécules correspond la forme de la courbe d'électrochimie (figure 6), indiquant globalement que des électrons ont été absorbés par le métal ? La question est donc de savoir comment, à partir d'une mesure molaire, collective, de molécules, il est possible de schématiser ce qu'il se passe pour les molécules individuellement. Supposant qu'il y a un fonctionnement et cherchant à l'élucider, les chercheurs sont amenés à imaginer ce que doit être le mode d'existence d'une molécule dans son milieu, avec les autres molécules, avec la surface si elle y est fixée ou adsorbée (déposée), en interaction avec le mouvement brownien. Le fait de déchiffrer un fonctionnement est une opération intellectuelle de schématisation, faisant coopérer l'imagination de l'objet individuel et les données quantitatives collectives des instruments de mesure.

Chercher à se rapporter au fonctionnement d'une molécule, c'est donc individuer la molécule. C'est le déplacement qui se produit lorsqu'on passe de la chimie supra- 
moléculaire ${ }^{25}$ aux machines moléculaires; même si les molécules produites peuvent être de même nature (caténanes, rotaxanes et dérivés), la démarche n'est plus exactement la même : à partir du moment où l'on appréhende les objets en termes de fonctionnement, on n'a plus une pluralité de molécules reliées par des liens «supra »: liaisons faibles, topologiques (nœud) ou mécaniques (anneaux entrelacés). On a une molécule dotée de fonctions, et plus seulement des molécules avec chacune des propriétés chimiques. Le thème de la machine dans les nanotechnologies signifie d'abord ce geste. Parler de machines n'est pas une métaphore mais correspond au fait d'individualiser les molécules. La molécule n'est plus un simple "morceau de matière ", constituant entrant dans la composition d'un matériau informé depuis notre échelle, mais directement une machine en action avec des fonctions.

21 Cependant, individuer la molécule ne veut pas dire simplement passer du collectif à l'individuel. Ce n'est pas forcément opérer avec une seule molécule. La philosophie de l'individuation de Simondon est précieuse pour cela : elle apprend à ne pas confondre l'individualité avec l'unité numérique ou l'identité substantielle. Quand du préindividuel s'individue, c'est toujours en individu et milieu associé. Plus une entité est individuée, et plus ses relations sont développées. Ce qui s'individue, c'est davantage une relation qu'une substance. Plus une molécule s'individue, plus son milieu associé lui est présent... pour le chercheur. Dans cette individuation technique se pose la question de la relation du chimiste avec ses objets. Dans la définition de "machine moléculaire » qui nous a été donnée au LCOM, la question du référentiel, c'est-à-dire du plan d'intelligibilité adopté, est incluse dans la définition: "une molécule ou une assemblée moléculaire multi-component avec signal externe qui déclenche un mouvement de grande amplitude de l'un ou plus des constituants alors que d'autres sont immobiles. Le référentiel reste à définir parce que ça bouge constamment ». La prise en compte de la relation entre les deux mondes, nano et macro, structure chacun des mondes. C'est en fait une individuation à deux (ou à trois avec le milieu), le milieu associé étant aussi ce qui associe le chercheur et la molécule. Le chercheur (co)opérant avec la molécule individuelle doit prendre en compte les relations de cette molécule avec les autres molécules. Ainsi sur la figure 7 une molécule est adsorbée (déposée physiquement mais non liée chimiquement) sur une surface. On voit que sa mise en mouvement (elle tournoie sous l'effet du mouvement brownien) dépend de sa proximité avec ses congénères. Il s'agit de la première image d'une rotation à l'échelle d'une molécule publiée obtenue avec un STM ${ }^{26}$. Les relations des molécules entre elles, tout comme les relations entre les chercheurs et des molécules, sont conditionnées par le type de milieu associé. Il en existe essentiellement deux types: la solution, milieu liquide encombré et chahuté par l'agitation thermique qui amène ses espèces à se mélanger dans tous les sens; la surface, stabilisée par l'utilisation de très basses températures et du vide, qui confère à la molécule un comportement quasi-classique par décohérence, mais où dominent les forces de surface. Le design des molécules-machines diffère selon le type de milieu qui va l'accueillir. Enfin le milieu associé est aussi le milieu de l'instrument, qui conditionne la façon dont on va échanger de l'information avec les molécules. L'idéal, disent les chimistes, serait de caractériser dynamiquement la molécule, mais c'est impossible ${ }^{27}$. Si le fait de "voir» (nous préférons dire "imager») ces molécules cristallisées par diffraction des rayons $\mathrm{X}$ coupe court à toute discussion sur leur figure, c'est alors le mouvement que l'on perd. Les dépôts sur surfaces permettent de caractériser les molécules individuellement au STM. Mais le mouvement est alors seulement déduit de 
mesures prises pas à pas. Le problème du dépôt sur surface est qu'il exige une autre chimie, avec des molécules fonctionnalisées précisément pour s'accrocher sur les surfaces. C'est pourquoi les molécules sur surface sont pour l'instant moins sophistiquées que leurs cousines en solution. Il y a donc une dialectique entre les méthodes de synthèse et les exigences de caractérisation dictées par les instruments. Cette dialectique informe le design des objets en agissant à son niveau par la constitution d'un milieu associé.

Figure 7: rotation d'hexa-butyl-deca-cyclene

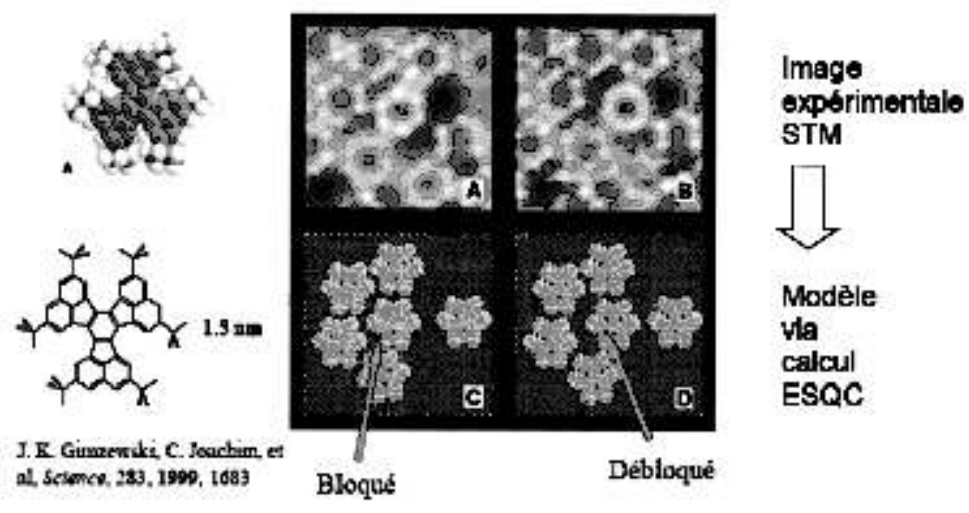

Source : présentation de Gwénaël Rapenne, CEMES

\section{Fonctionnaliser le milieu associé}

Au Laboratoire de PhotoPhysique Moléculaire (LPPM), le groupe de Gérald Dujardin a réalisé, après des aléas d'un projet de circuit logique moléculaire, une molécule bistable activée électroniquement au STM sur une surface (figure 8). Quand la molécule est impulsée par la pointe d'un STM, on constate que le pulse se traduit par une rotation de la molécule sur un de ses groupements phényls. Il ne s'agit pas d'un mouvement mécanique, mais de deux stations électroniques occupées par la molécule. Les chercheurs du groupe entretiennent autour de cette manipulation un débat pour savoir ce qu'il faut fonctionnaliser : molécule ou surface? Ce débat a lieu entre le chercheur qui étudie et prépare la surface et celui qui dépose la molécule. Alors que celui qui s'occupe des molécules insiste sur la nécessité de fonctionnaliser la surface, sur le fait que "la surface est une partie de la machine ", celui qui s'occupe des surfaces insiste quant à lui sur l'importance de la fonctionnalisation des molécules (certainement parce que pour lui, la molécule doit servir à explorer la surface, considérée comme ce qu'il faut connaître). Dans les deux cas la molécule et/ou la surface doivent être fonctionnalisées de telle manière que la surface soit une fonction de la machine. Ce qu'il s'agit de fonctionnaliser, c'est le milieu associé. On a dans ce débat les linéaments d'une démarche d'invention: rendre compatibles des réquisits contradictoires pour reformuler le problème technologique qui a présidé à la réalisation de l'objet. Par exemple, ils émettent une hypothèse sur les fonctions de la surface : sa fonction, ce serait de "ralentir la nanomachine ", de lui conférer un comportement quasi classique, et non seulement quantique. La démarche est une démarche de connaissance mais elle concerne des fonctionnements technologiques (comprendre la relation de l'environnement et du fonctionnement de la machine dans un milieu associé). C'est une 
connaissance de l'individuel : chaque machine est spécifique à son environnement; certaines mesures sont faites sur une molécule et la même. Cependant cela n'empêche pas cette démarche de poursuivre une finalité générique. Comme chez Simondon, il s'agit de fonctionnaliser le milieu associé pour pouvoir se passer de la dépendance à l'égard d'un milieu déterminé. Telle est bien l'idée de Christian Joachim, coordinateur du groupe nanosciences du Centre d'Élaboration des Matériaux et des Systèmes (CEMES) : aider la molécule à conquérir son «indépendance machinale ». Il s'agit en somme de travailler au caractère transposable ou reproductible du singulier. Les chercheurs n'en sont certes pas là ; ils se disent encore "à l'Âge de pierre »; cependant, certains d'entre eux affirment qu'il est déjà tellement extraordinaire d'échanger de l'information avec une molécule individuelle, de regarder « les yeux dans les yeux » un comportement probabiliste, qu'il est absurde d'en demander plus, tant les possibilités d'application sont lointaines, indéfinies... et peu intéressantes pour un chercheur.

Figure 8 : biphényl bistable sur surface de silicium

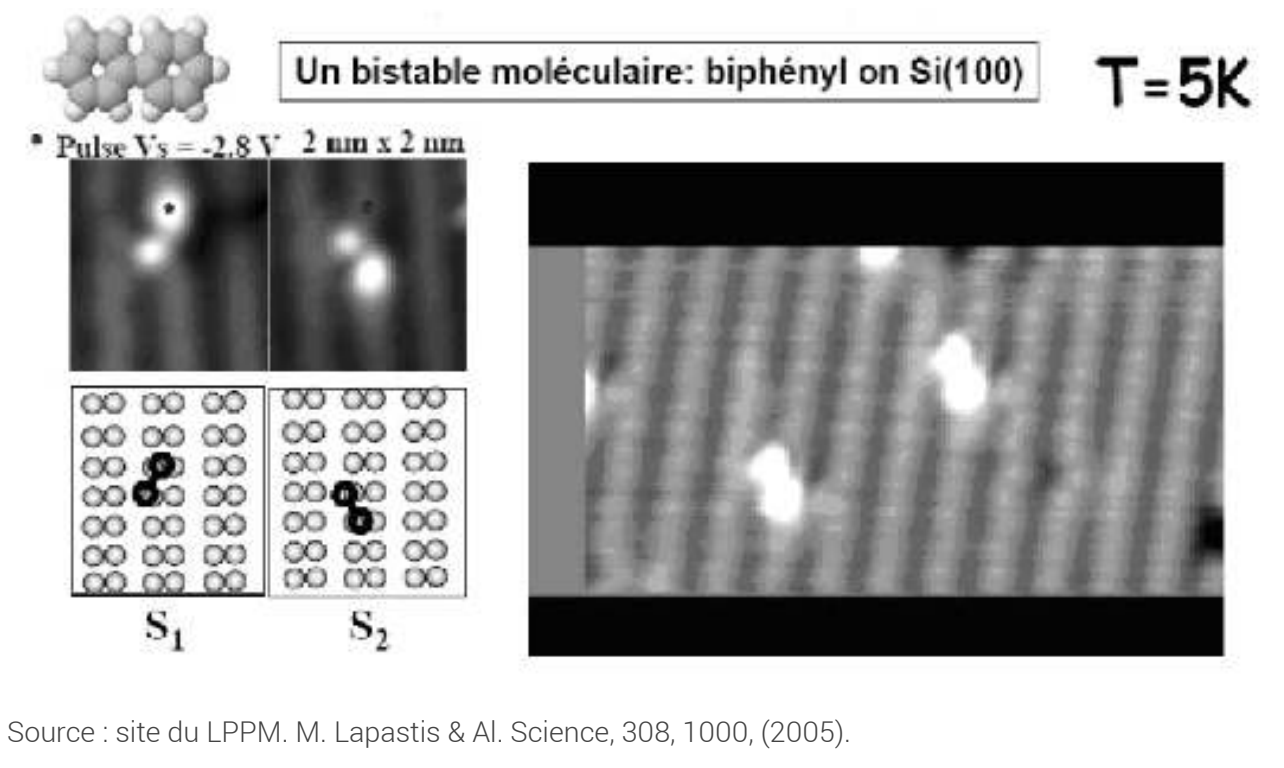

\section{Du molaire au moléculaire : cartographier}

En raisonnant en termes de fonctions sur des molécules singulières, ce sont les rapports entre le molaire et le moléculaire qui changent. En effet, le moléculaire n'est plus appréhendé par le seul molaire, comme c'est le cas traditionnellement en chimie. Comme le dit Christian Joachim, la chimie est une sorte de"thermodynamique assistée "; elle n'appréhende le moléculaire qu'à travers sa représentation molaire. C'est pour cette raison que les chimistes utilisent le vocabulaire de l'«assemblée " moléculaire : il s'agit de représentation au sens politique. Une partie des molécules, la part de la réaction chimique qui réussit, c'est-à-dire qui se conforme aux prévisions du plan de synthèse, est "élue" pour représenter la majorité. L'assemblée est le type de processus que le chimiste élit, c'est-à-dire choisit de surcoder comme majoritaire, même si elle ne représente qu'une partie des molécules en solution, celles qui entrent dans le taux du rendement ${ }^{28}$. Les formules chimiques sont des instantanés prélevés sur les processus moléculaires pour les besoins de la prévision: comment enchaîner les réactions pour avoir au final la molécule désirée ? La partie surcodée est caractérisée, 
isolée, purifiée, reproduite, puis engagée dans de nouvelles étapes de synthèse, et ainsi de suite. Le reste est mis de côté. Le chimiste, traditionnellement, ne s'occupe que des lignes surcodées, il décalque, il enchaîne des fascicules. La schématisation du fonctionnement met en relation l'art de la synthèse chimique avec d'autres démarches intellectuelles et pratiques. Loin de prétendre s'y substituer, elle le rapproche de son objet. Si le physicien peut encore voir, selon les préjugés longtemps en vigueur, le chimiste comme sa petite main industrieuse, le chimiste est responsable du design de la molécule, aussi est-il libre le modifier en cours de route ou d'exercer son droit de veto, si le physicien lui demande des molécules inaccessibles. Sur le plan épistémologique, la chimie des machines déplace l'intérêt exclusivement accordé au "surcodage " des formules chimiques vers le «codage-décodage » constant de la «vie» des molécules. C'est seulement après être passé par ces lignes décodées que l'on revient aux logiciels de surcodage, formules et modèles. C'est la différence entre la décalcomanie et la cartographie. Celui qui décalque s'abstrait du milieu, se réfugie au-dessus. Celui qui cartographie doit s'orienter stratégiquement parmi les lignes et les singularités du plan. Le chimiste entre en quelque sorte dans la solution, n'est plus au-dessus de son objet mais bien à ses côtés. Il s'attache à la manière dont les molécules, individuellement, «font des bêtises », comme le dit Jean-Pierre Sauvage. Le geste opéré s'apparente donc au geste de la cartographie, comme le décrivaient Gilles Deleuze et Félix Guattari dans Mille Plateaux : non plus seulement « décalquer » mais » reporter le calque sur la carte ».

Figure 9 : L-Lander sur surface de cuivre $\mathrm{Cu}(100)$

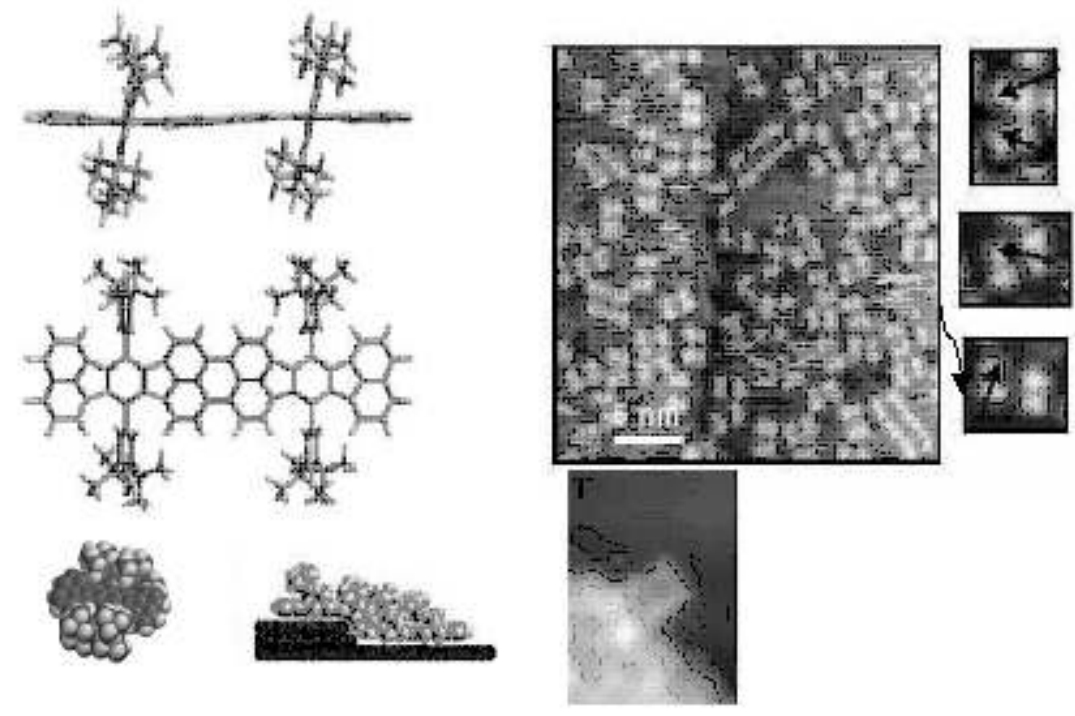

À gauche : modèles (« fil de fer » et dynamique moléculaire) ; à droite : images STM

Source : site Internet du CEMES http://www.cemes.fr

Le lander moléculaire du CEMES (figure 9) fournit un exemple intéressant de cette production de connaissance par des «machines à cartographier » dont la vocation est exploratoire : le lander a été muni de "pieds » destinés à isoler électroniquement le plateau de la surface; à cause de cela, on n'image que les pieds et non le plateau de la molécule. Ainsi isolé, le plateau peut servir de «fil amovible " pour connecter le niveau supérieur de la marche d'une terrasse avec la pointe du STM. Elle devient alors une «machine-dispositif » : elle permet de prendre une mesure qui n'aurait jamais pu être 
effectuée autrement. Résultat inattendu (en bas): on constate que la molécule se déforme tout en déformant le pas de la terrasse. Tout se passe comme si le sujet et l'objet s'échangeaient: le physicien, traditionnellement derrière son instrument, est comme transporté sur la surface ; la molécule, en principe examinée sous la pointe du STM, devient ici une pièce clé du dispositif expérimental lui-même.

L'aspect cartographique explique en partie le leitmotiv de l'emploi de l'expression «nanomonde »: il s'agit de faire de l'échelle nano un monde en dressant des cartes (voir la figure 10). Les machines, qu'elles soient objets cartographiés et/ou dispositifs cartographiant, sont censées qualifier ce monde en y développant une technè. En faisant fonctionner (ou dysfonctionner) ces artefacts, nous sommes mis en relation avec des phénomènes physiques avec lesquels nous n'étions pas en relation avant. Loin de supprimer la polarité entre nature et artifice, ces pratiques approfondissent la polarité sans opposer les deux pôles.

Figure 10 : cartographier une surface et ses habitants

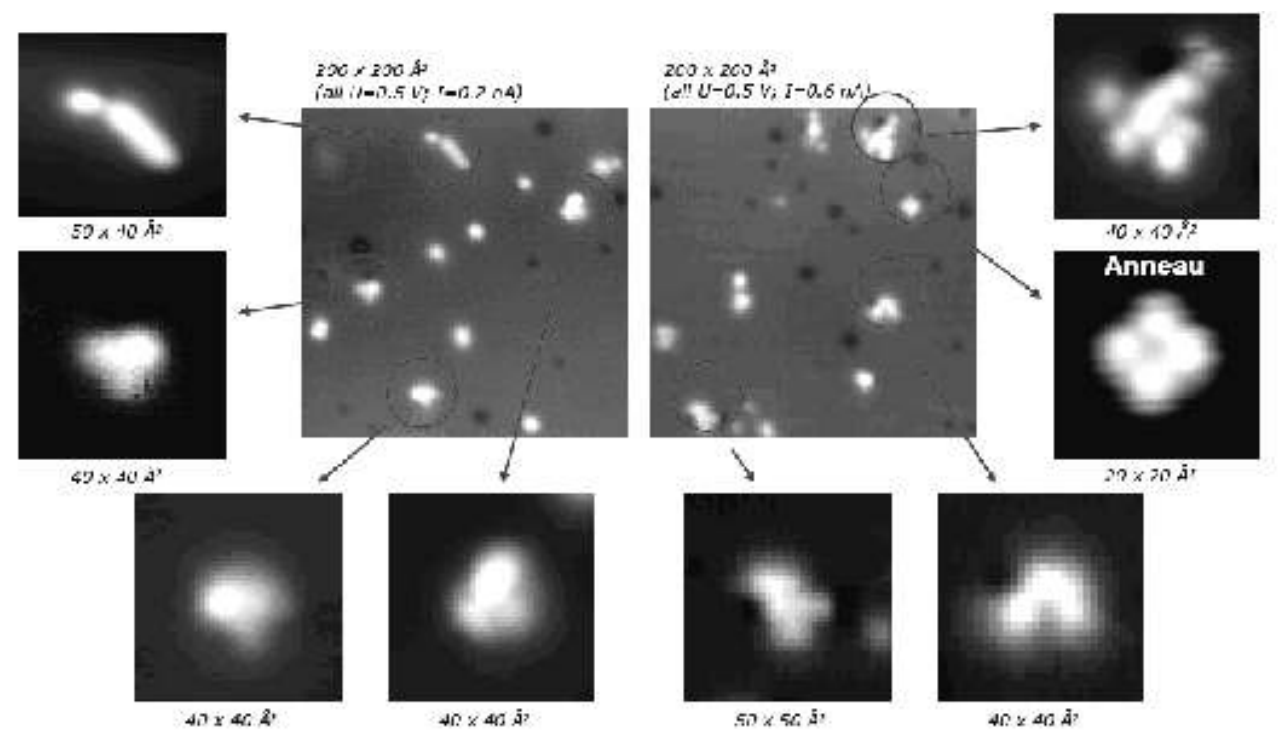

Source : extrait d'une présentation de Gwénaël Rapenne, CEMES

Rapennne, G. " Vers la miniaturisation ultime et le contrôle de la rotation à l'échelle moléculaire ", Du métal aux nanotechnologies, ASPROM-UIMN, Paris, 17-11-2006. Il s'agissait dans cette expérience d'aller contacter une molécule déposée sur la surface. Les chercheurs constatent que la molécule a été disloquée pendant la phase d'« atterrissage » en un certain nombre de sous-produits. « Un vrai zoo!» s'exclame le chimiste.

Dans cette pratique de "mapping", le savoir est une distribution entre des connaissances de l'individuel (des fonctionnements singuliers et des tables de données quantitatives locales) et des relevés topographiques (types de surfaces, en fonction de la température, de la pression, etc.). Tout ce petit monde semble fonctionner moins par application pratique de connaissances théoriques que par concrétisations d'objets individuels reliés par les lignes du nanomonde qui se dessine autour d'eux. Les théories sont omniprésentes (physique mésoscopique, chimie quantique, physique des surfaces, computation quantique, matière condensée, confinement, électromagnétisme, optoélectronique, transfert d'électron, électrodynamique, théories d'instruments, etc.), 
mais elles sont engagées dans les pratiques soit au titre de «background knowledge » appartenant à un arrière-plan disciplinaire (« en optoélectronique on m’a appris qu'un photon interagissant avec un électron... »), soit au titre d'instrument prévisionnel. Elles sont de plus en plus nécessaires et de moins en moins suffisantes. Elles tendent de plus en plus à s'identifier en physique à ce qu'elles sont en chimie: des modèles qui fonctionnent ${ }^{29}$. Le modèle importe en ce qu'il fournit un plan d'intelligibilité dans l'accès à l'objet, une manière de le visualiser, ou de prévoir son fonctionnement. Mais ce que notre "machiniste moléculaire " cherche à connaître, c'est bien plutôt la machine concrète et ce qu'elle peut, son mode d'existence, en deçà des théories dont chacune fournit un plan d'intelligibilité de l'objet. Les mêmes machines moléculaires, qui servent au chimiste à parfaire l'art des synthèses et la connaissance des molécules, servent au physicien à tester la physique quantique. Chacun affine donc ses concepts et ses méthodes. Mais l'objet déborde les partitions disciplinaires et ne semble se réduire à aucun de leurs objectifs pris séparément. L'objet n'est pas déductible d'une seule théorie, il les fait toutes fonctionner en même temps.

Ainsi se développe un savoir technologique des fonctionnements à la nano-échelle. Il connecte entre elles des connaissances individuelles en dressant des cartes. Tout cela peut ressembler à une entreprise de colonisation de la matière à l'échelle moléculaire, qu'il s'agirait de faire travailler à notre service. Mais plutôt que d'une entreprise de colonisation et de maîtrise, nous préférons voir les nanotechnologies comme une science de terrain pratiquée en laboratoire ou inversement comme une technologie de laboratoire en plein air (médiatique). Ainsi Christian Joachim considère-t-il ses landers comme des sondes envoyées sur Mars, avec phase de lancement, phase d'exploration, etc. (figure 11). Le postulat méthodologique d'une science de terrain est le refus de plaquer par avance une grille interprétative théorique sur les singularités du terrain exploré. Son principal écueil est le cliché héroïque et viril de l'exploration d'un Far West - «à la conquête du nanomonde »! Toute exploration par des machines équivautelle à une colonisation? Cherche-t-on à convertir et à soumettre "les indigènes » où plutôt à les rencontrer pour faire alliance avec eux ? Ces questions restent ouvertes et doivent le rester, malgré et parfois contre la rhétorique des grands discours, mais elles doivent être posées. Si l'entreprise s'oriente vers une volonté de maîtrise inconditionnelle de la matière par l'homme, elle échouera forcément et se montrera ou décevante, ou dangereuse, victime de ses illusions de contrôle.

Figure 11 : les landers sur Mars

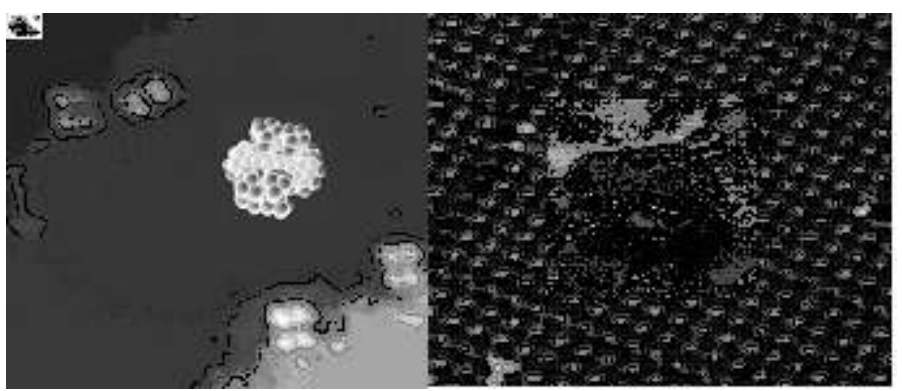

Source : montage réalisé à partir d'une présentation de Christian Joachim, CEMES.

Science ou technique ? La question présuppose que s'il s'agit de science, les chercheurs peuvent faire ce que bon leur semble et que, s'il s'agit de technique, les finalités devront être clairement énoncées pour que l'on sache au moins à quoi s'attendre. Or 
ces recherches ne sont menées ni pour résoudre des problèmes théoriques ni pour résoudre des problèmes immédiatement pratiques. Il faudrait donc s'abstenir de les juger depuis les critères de «la Belle Science » dominée par l'ordre prétendument pur du théorique et « la volonté de faire science ». Il ne faudrait pas non plus projeter sur elles une caricature de technologie, réduite à de l'utilitaire. Il serait plus judicieux d'accorder un statut épistémologique propre à la connaissance technologique - ou tout simplement techno-logie - ; une fois que l'on aura admis qu'elle n'est ni du théorique déchu, ni de l'utilitaire masqué, mais qu'elle consiste en des modes d'intelligibilité propres, on pourra discuter de ses valeurs.

\section{Se référer à la nature dans les failles du fonctionnement}

Selon Jean-Pierre Sauvage, le fait même de parler de machines est venu de la biologie. C'est la mise en évidence en 1997 par des biophysiciens, Yoshida et Kinosita, de la rotation de l'ATP synthase qui a définitivement "donné du corps" au concept de machine à l'échelle des molécules ${ }^{30}$. L'ATP synthase est une enzyme universelle au vivant eucaryote et procaryote, présente dans les membranes des mitochondries, quasiment similaire d'une espèce à l'autre. Enzyme indispensable au cycle métabolique de la « respiration cellulaire », elle fournit le « fuel » de la cellule, l'Adénosine TriPhosphate (ATP), dont elle produit chaque jour l'équivalent en poids de l'organisme, à partir d'ADP + Pi (adénosine diphosphate + phosphate inorganique). En présence d'une différence de potentiel électrochimique induite par la différence de concentration en ions $\mathrm{H}^{+}$de part et d'autre de la membrane, des protons traversent la membrane, entraînant un barillet (figure 12). Une tige tourne alors dans la tête de l'ATP synthase pour séparer les sous-unités $\alpha$ et $\beta$, libérant ainsi l'ATP des sites catalytiques. Inversement, en l'absence de gradient électrochimique et en présence d'ATP, la molécule est capable d'hydrolyser (de «casser ») la molécule d'ATP en ADP + Pi pour recréer une différence de potentiel de part et d'autre de la membrane; elle est alors dite "ATPase " ou " pompe à protons »; la canne tourne alors dans l'autre sens. Le fonctionnement est donc réversible. Elle a soit une activité de catalyse, soit une activité d'hydrolyse. Connue depuis longtemps, sa rotation a été mise en évidence expérimentalement par Yoshida et Kinosita en 1997. Ils l'ont implantée «la tête en bas » sur une surface artificielle et lui ont greffé une protéine filamenteuse d'actine fluorescente assez longue ( 2 microns) pour que l'on puisse visualiser sa rotation par microscopie à fluorescence. 


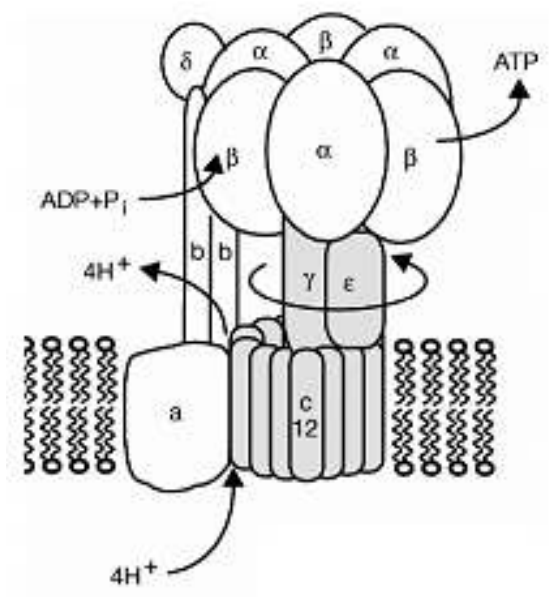

Sauvage, J-P. : « Les nanomachines moléculaires, de la biologie aux systèmes artificiels et aux dispositifs », L'actualité chimique, 2003, 119-125.

Source, Rastogi \& Girvin, Nature, 1999

31 Avant 1997, les chimistes parlaient, pour les mêmes objets, de «systèmes dynamiques ". Il y avait quelque chose dans la nature qui répondait à ce que l'on faisait artificiellement. Jean-Pierre Sauvage y a vu une grosse rotaxane, certes complexe et plus élaborée, mais bien analogue à celles qu'ils synthétisaient depuis une décennie au LCOM. Désormais, il était reconnu que les rotaxanes, ces objets chimiques exotiques et inutiles, existaient dans la nature. Depuis, même si toutes les molécules du LCOM sont issues d'une accumulation progressive de méthodes et de designs, d'un savoir-faire relevant de l'artifice, les chercheurs prétendent constamment s'inspirer de la nature. Ainsi, beaucoup de leurs machines moléculaires trouvent un correspondant biologique : les protéines chaperons, la membrane du virus HK97, les moteurs protéiques linéaires assurant le glissement des fibres du muscle strié. Or les chimistes le disent clairement : contrairement à ce qu'on peut lire dans les publications, c'est toujours après coup que l'on cherche dans la biologie à quoi telle ou telle machine moléculaire synthétisée peut bien ressembler. Par exemple le design du muscle moléculaire (figure 13) n'est pas inspiré des protéines actine-myosine comme cela est dit (figure 14), mais vient du dysfonctionnement heureux d'une autre machine moléculaire, synthèse ratée qui s'est révélée féconde grâce à l'ingéniosité des chimistes. 


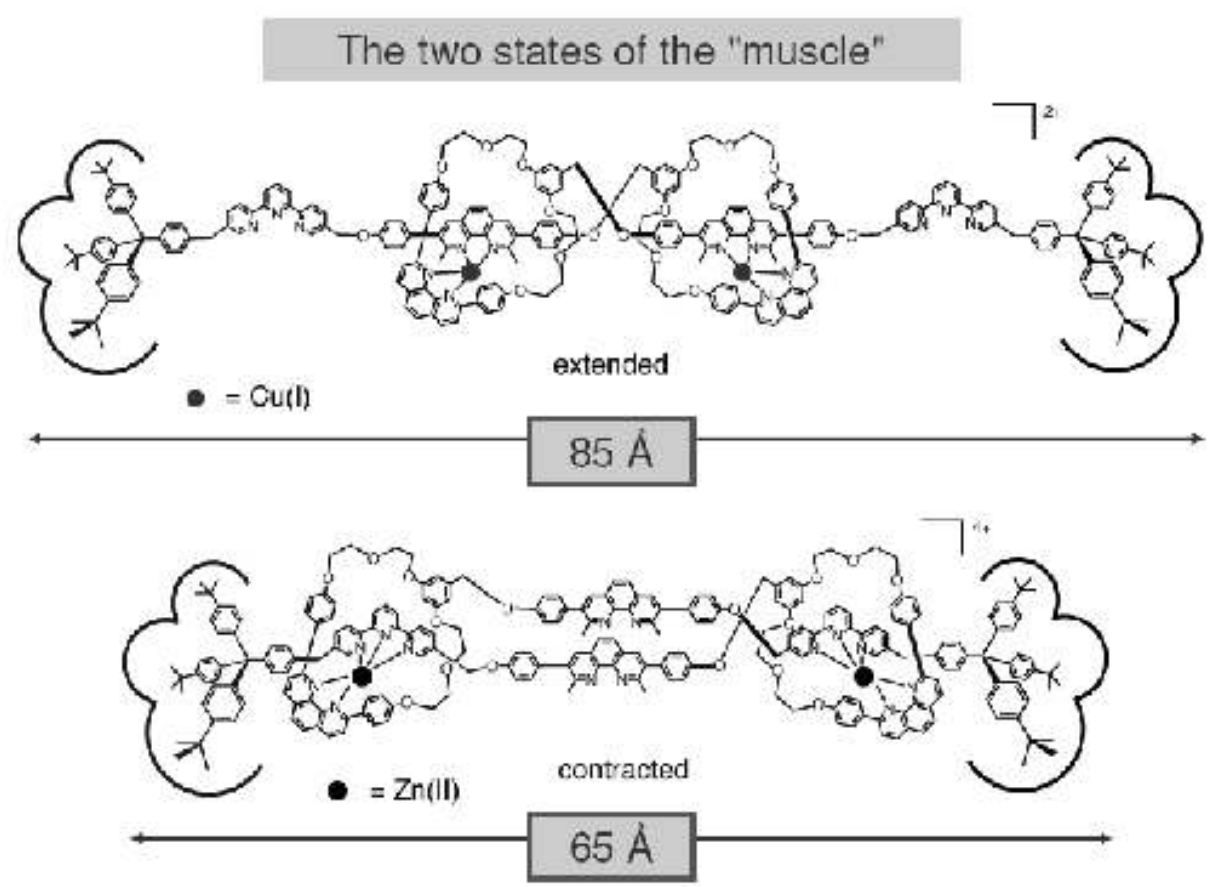

Source : extrait d'une présentation de Jean-Pierre Sauvage, LCOM

Figure 14 : Les protéines du muscle moléculaire naturel

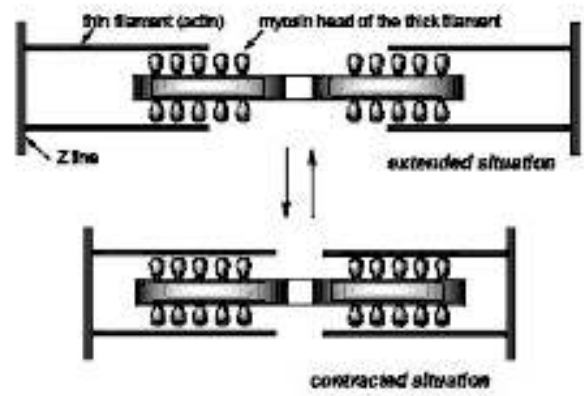

Représentation très schématique du fonctionnement du muscle strié : le glissement du filament fin le long du filament épais conduit à contracter ou allonger le muscle. Source : Sauvage, J-P. : « Les nanomachines moléculaires, de la biologie aux systèmes artificiels et aux dispositifs ", L'actualité chimique, 2003, 119-125.

Même si elle permet de «faire passer » une publication et d'attirer ainsi l'attention sur un objet exotique, inédit et bizarre en le rattachant à quelque chose qui existe déjà, la référence à la nature n'est pas considérée par les chimistes comme un simple fairevaloir. Si la nouveauté ne peut être reconnue qu'en éclairant rétrospectivement l'ancien, il faut voir ce processus comme une manière de donner du sens à ces recherches. Les recherches sur les machines moléculaires artificielles sont par là une manière de développer des intuitions technologiques pour une connaissance des machines non-vivantes du vivant (telle l'ATP synthase). Cette donation de sens ne se fait pas par une identification simple du naturel et de l'artificiel, mais par une référence a posteriori à la nature : en fabriquant un artefact on apprend quelque chose de l'ordre du fonctionnement sur la nature, par exemple le rapport qu'il peut y avoir 
entre le transfert d'électrons dans la molécule et la forme de celle-ci, ou la question du rôle "cliquet" de l'effet de taille dans le passage d'une rotation quantique, sans direction privilégiée, comme sur la figure 7, à une rotation classique comme dans l'ATP synthase, directionnelle, mais pourtant réversible (son mécanisme demeure un mystère). Dans les nanotechnologies, analogies machiniques et démarches de réingénierie interviennent sur des objets précis pour lesquels les modèles et les cadres d'application d'un certain nombre de théories ne permettent d'en appréhender que des facettes dissociées. Ainsi pour les machines à base de protéines du vivant, les modélisations par réactions en chaîne des biochimistes et les modèles probabilistes des biophysiciens sont incompatibles; chacun décrit théoriquement une facette du fonctionnement, mais aucun ne permet de l'élucider entièrement. Le chercheur sera alors satisfait s'il aura réussi à modifier l'objet pour l'insérer dans un dispositif de visualisation de celui-ci en action.

Les chimistes du Laboratoire d'Électrochimie Organique et de Photochimie Rédox (LEOPR) sont formels : on n'imite pas le vivant en tenant compte des ressemblances, on opère des analogies fonctionnelles avec lui. Il ne faut pas comprendre le «biomimétisme " comme une pure et simple imitation du biologique. Le vivant, ou plutôt les machines qui participent à son métabolisme sont considérées comme des normes technologiques (c'est tout l'objet du livre de Richard Jones, Soft Machines, Nanotechnology and Life). Parfaitement intégrées à leur milieu, ayant cessé d'évoluer ${ }^{31}$, ce sont pour nos chimistes devenus technologues des machines parfaitement concrètes, mais qui n'ont pas fait l'objet d'une démarche d'invention. Ce sont des machines concrètes sans processus de concrétisation. Pour cette raison, leur reconstruction et leur modification atome par atome sont probablement inaccessibles à la technologie humaine, qui suppose un certain degré d'abstraction et un temps relativement court par rapport au temps biologique: celui de la formulation d'une intention donnant lieu à un problème technique que la démarche d'invention tente de résoudre par concrétisation. C'est la différence entre les contextes et les temporalités de production naturels et artificiels qui comptent ; le temps segmentarisé et linéaire de la synthèse chimique est toujours à la fois trop long et trop court par rapport aux temporalités complexes et enchevêtrées de l'évolution biologique; trop long par rapport à la complexité des molécules naturelles (il faut déjà plusieurs années pour synthétiser une machine moléculaire artificielle très simple), trop court par rapport au temps global de l'évolution. Ce sont deux individuations différentes, ce qui ne veut pas dire qu'il n'y ait pas de passerelles analogiques entre les deux. Les machines du vivant sont prises dans des analogies de fonctionnement; nos machines macroscopiques aussi ; mais le centre de l'analogie, son support ou son "modèle», c'est la machine artificielle, celle qui est inventée par les chimistes, se rendant possible sans autre modèle qu'elle-même dans un processus de concrétisation technique. La référence constante à la nature dans les pratiques ne rabat jamais l'un sur l'autre le naturel et l'artificiel comme le fait la rhétorique des grands discours, qui transforme la relation d'analogie en relation d'identité. Leur différence comme leur relativité est fondée technologiquement, sans qu'il y ait besoin de recourir à une différence d'ordre métaphysique ou théologique. 


\section{Conclusion}

Comment passe-t-on des pratiques bien circonstanciées que nous venons de décrire aux grands discours rapportés dans la première partie? Un discours hype sur la technologie, c'est une manière d'articuler dire et voir, aurait dit Foucault. Mais une pratique technologique de laboratoire aussi, c'est une manière de parler de ce que l'on voit et de produire des visualisations de ce dont on veut parler. Il y aurait alors à la membrane des pratiques et des grands discours des zones d'indétermination du rapport entre dire et voir. Ces zones d'indétermination sont celles où le discours peut dire à la fois la même chose et autre chose que ce que montre la pratique. Il s'agit du point où les pratiques donnent prise aux grands récits évoqués dans la première partie, tout en ne coïncidant pas avec eux. Se produit alors ce déphasage entre les pratiques singulières et contextualisées et les grands discours. Dans ce que nous avons décrit, nous pouvons déceler trois zones d'indétermination où se produit ce déphasage. À chaque fois, une polarité qui a du sens dans la pratique se transforme en une exténuation: la référence analogique à la nature depuis les failles du fonctionnement des artefacts devient une exténuation de la polarité nature-artifice ; la relativisation de l'opposition sujet/objet dans ces pratiques devient exténuation du statut de l'objet et exacerbation du pôle anthropologique dans la figure d'un sujet-objet de consommation; enfin l'abandon de l'idéal de représentation dans la pratique scientifique devient une stratégie de cryptotechnicité minant notre relation aux objets techniques.

Nous avons donné assez d'éléments pour le premier. Contentons-nous d'évoquer les deux autres : ce qui induit cette exacerbation du pôle anthropologique, c'est la mise en question du statut d'extériorité ontologique de l'objet, tel qu'il était pensé dans les sciences modernes, par opposition au sujet épistémologique de la connaissance. Le rapport cognitif à l'objet est ici connaissance par participation. Cette relation est investie d'une grande part d'affectivité de la part des chercheurs, qui bien souvent s'identifient de manière sensori-motrice avec le fonctionnement de l'objet dans le dispositif. C'est pourquoi les machines moléculaires ne sont pas tout à fait des objets au sens où elles seraient dominées par le sujet de la connaissance; ce sont bien des objets au sens où il y a un processus d'objectivation, auquel concourt toute une série de médiations instrumentales qui rendent consistante et résistante cette matière qui fonctionne, mais les objets ne sont pas opposés au sujet. » Imaginons que vous êtes un électron, dit Christian Joachim, comment passez-vous à travers une molécule?» Simondon dans le cours Invention et développement des techniques ${ }^{32}$, met bien en valeur cette dimension relationnelle affective de l'objet individualisé :

Ces aspects relationnels complexes ont une grande importance parce qu'ils situent l'ustensile ou l'appareil précisément au même niveau que l'opérateur ou l'utilisateur; l'objet technique individualisé [c'est-à-dire ni l'élément, ou l'outil dominé par le schéma corporel du vivant, ni l'ensemble ou le réseau qui est plus grand que l'individu s'y trouvant inclus] est ce que rencontre l'homme, en tant que partenaire fonctionnel, dans son travail et au cours de son existence [...] ils se chargent d'une signification culturelle considérable (lampe, horloge); ils sont les socii de l'existence ${ }^{33}$.

Exténuation de l'objet traditionnellement opposé au sujet de la connaissance, les nanotechnologies ne signifient pas pour autant l'exténuation de tout objet, mais sa sortie du régime d'objectivité classique. 
Enfin l'enrôlement des objets dans les discours futuristes les rend cryptotechniques (troisième déphasage), et il est induit par l'abandon de l'idéal et des dispositifs de la représentation dans l'imagerie scientifique. Le STM est paradigmatique de ce déplacement: il image en manipulant; produites par toucher, en interagissant avec le champ proche de l'objet, les images STM ne sont plus des re-présentations à distance de l'objet - cette distance où s'insère l'explication, longtemps considérée comme un trait distinctif de l' ethos du scientifique - mais sont à même l'objet. L'image n'est plus la projection de certains aspects de l'objet sur une surface d'inscription, elle devient une membrane de l'objet. Adhérant à l'objet comme une seconde peau, ces images-membranes inaugurent des possibilités indéfinies de changement de peau des objets, de déguisement en déguisement. Les exemples de ce type sont légion et font partie du régime de production et de diffusion du savoir très particulier des nanotechnologies. Ainsi notre biphényl bistable (figure 8) a-t-il été nommé « boutons de contrôle picométrique » pour le service de communication du CNRS. On peut y lire que « ces dispositifs, permettant de distribuer des informations optoélectroniques à l'échelle nanométrique, pourraient servir de « cerveaux » aux futures machines moléculaires ${ }^{34}$ ». Or cela ne peut être vrai que si l'on abstrait l'objet du dispositif qui permet son imagination (sa mise en image) : l'énorme STM, les basses températures, le choix de la surface, le dispositif d'amplification, les logiciels associés, etc. Cela ne veut pas dire qu'il faille en appeler à un retour de la froide distance de la représentation, puisque de toute façon, les dispositifs techniques d'imagination "nano" n'en relèvent plus, mais qu'il y a à reconnaître tout un régime des images-objets pour y discerner de nouvelles modalités d'intelligibilité. Car c'est là que se situe finalement la zone obscure des nanotechnologies. Nous disions que ce sont les pratiques qui sont voilées par les discours. Mais il ne suffit pas de lever le voile pour faire la lumière. Ce qui est obscurci, dans les pratiques comme dans les discours, c'est le rapport que ces objets et nous entretenons à l'image.

\section{BIBLIOGRAPHIE}

Drexler Éric K., Engins de Création, l’avènement des nanotechnologies [1986], Paris, Vuibert, 2005.

Guchet Xavier, Les sens de l'évolution technique, Paris, Léo Scheer, 2005.

Joy Bill, « Why the future doesn't need us? », Wired, Issue 8.04, avril 2000, http:// www.wired.com/wired/archive/8.04/joy.html

Kapp Ernst, Grundline einer Philosophie der Technik, traduction, Principes d'une philosophie des techniques, Paris, Vrin, 2007.

«La nanotechnologie : l'innovation pour le monde de demain », RDT Info, Commission Européenne.

Moore George E., Principia Ethica, Cambridge, Cambridge University Press, 1903. 
Nerlich Brigitte, «From Nautilus to Nanobo(a)ts: The Visual Construction of Nanoscience », AZojono, 2005, http://www.azonano.com/details.asp?ArticleID=1466

Nordmann Alfred, « Ignorance at the heart of science? Incredible Narratives on Brain-machine Interfaces », 2006. http://www.uni-bielefeld.de/(en)/ZIF/FG/2006Application/PDF/

Nordmann_essay.pdf

Rees Martin, Our Final Century, Londres, Heinemann, 2003.

Roco Mihail C., Bainbridge William Sims, Converging Technologies for Improving Human Performance, Nanotechnology, Biotechnology, Information Technology and Cognitive Science, rapport NBIC, NSF/DOCsponsored report National Science Foundation, Arlington, Virginia, Juin 2002, http:// www.wtec.org/ConvergingTechnologies/1/NBIC_report.pdf

Roukes Michael, «Plenty of room indeed », Scientific American, 2001, p. 42-49.

Simondon Gilbert, « Psychosociologie de la technicité », Bulletin de l'École pratique de psychologie et de pédagogie de Lyon, $\mathrm{n}^{\circ}$ 2, novembre-décembre 1960, p. 127-140; $\mathrm{n}^{\circ}$ 3, janvier-février 1961, p. 227-238; n ${ }^{\circ}$, mars-juin 1961, p. 319-350.

Simondon Gilbert, L'Individu et sa genèse physico-biologique, Paris, Aubier, 1989.

Simondon Gilbert, Invention et développement des techniques, Paris, Seuil (Traces écrites), 2002.

Yoshida Masasuke, Noji Hiroyuki, Yasuda Ryohei, Kinosita Kazuhiko JR, « Direct observation of the rotation of $\mathrm{F}_{1}$-ATPase ", Nature, $\mathrm{n}^{0} 386,1997$, p. 299-302.

\section{NOTES}

1. Gilbert Simondon, L'Individu et sa genèse physico-biologique, Paris, Aubier, 1989, p. 44.

2. Les nanotechnologies constituent un champ de recherches flou et englobant, difficile à circonscrire. Elles peuvent se définir comme l'ensemble des pratiques qui étudient et manipulent la matière au niveau de ses constituants atomiques et moléculaires, visant à tirer parti des propriétés et des comportements spécifiques de la matière à cette échelle. Regroupant de nombreuses disciplines scientifiques et d'ingénierie, elles forment une vaste nébuleuse qui ne comprend pas que des pratiques forcément nouvelles. Certaines se sont re-labellisées "nano- » pour bénéficier du vent favorable venant des bailleurs de fonds. On se focalisera ici sur un champ de recherche relativement restreint et délimité, celui des nanomachines moléculaires artificielles, car ses praticiens y revendiquent souvent un changement dans l'ingénierie et ses concepts. Par ailleurs il se prête plus aisément que d'autres domaines à une utilisation opératoire des concepts de Simondon, parce qu'il est en puissance d'objets individualisés qui fonctionnent, c'est-à-dire en puissance de machines. Nous n'excluons pas cependant les autres domaines. Simplement, nous considérons les recherches sur les machines moléculaires comme relativement paradigmatiques du champ des nanotechnologies, car le vocabulaire machinique est omniprésent dans toutes ces pratiques.

3. La critique du schème hylémorphique est l'une des voies d'entrées possibles dans la philosophie de Simondon. Nous la supposons donc connue. Rappelons simplement que Simondon décrit le schéma hylémorphique comme une des deux grandes réponses traditionnelles à la question de l'individuation (la première étant la voie substantialiste). La matière reçoit la détermination d'une forme individuelle ou bien oppose la résistance de ses particularités à une forme spécifique: cela donne un individu. Contre cette habitude de pensée, Simondon veut essayer de décrire l'individuation en dehors de l'opposition de la forme et de la matière. 
4. Dans le cadre d'un travail d'enquêtes et d'entretiens de terrain menés avec Xavier Guchet au sein du groupe ANR Bionanoethic, coordonné par Bernadette Bensaude-Vincent.

5. Extrait d'une brochure educative, UK's National Physical Laboratory (notre traduction).

6. «La nanotechnologie: l'innovation pour le monde de demain», RDT Info, Commission européenne. www.cordis.lu/nanotechnology

7. Référence aux Principia Ethica de G.E. Moore, Cambridge University Press, 1903. Moore nomme «sophisme naturaliste» ou «natural fallacy» l'argument des philosophes hédonistes qui identifient le bien au plaisir et justifient cette assertion en montrant que la recherche du plaisir est naturelle. Moore montre que leur erreur est de confondre le descriptif avec le normatif en affirmant que ce qui est bien est naturel et que ce qui est naturel est bien.

8. Tel est le sens de la critique que Simondon adresse aux cybernéticiens quant à la pertinence de la figure de l'automate pour articuler le régime du vivant et le régime technique.

9. Non pas autonome au sens où il se ferait sans nous, mais ayant ses déterminations et des conditions d'intelligibilité propres. On consultera à ce propos le livre de Xavier Guchet, Les sens de l'évolution technique, Paris, Léo Scheer, 2005.

10. On consultera le rapport NBIC, Converging Technologies for Improving Human Performance,Nanotechnology, Biotechnology, Information Technology and Cognitive Science, M.C. Roco, W.S. Bainbridge, NSF/DOC-sponsored report National Science Foundation, June 2002, Arlington, Virginia, http://www.wtec.org/ConvergingTechnologies/1/NBIC_report.pdf

11. Selon Leroi-Gourhan, l'homme est cet être toujours déjà extériorisé. Rappelons que tout un courant de la philosophie des techniques identifie le développement des techniques à un processus d'extériorisation des fonctionnalités et des capacités du corps. Une "projection organique ", selon la conceptualité d'Ernst Kapp. Voir Ernst Kapp, Grundline einer Philosophie der Technik, traduction, Principes d'une philosophie des techniques, Paris, Vrin, 2007.

12. C'est-à-dire tout ce par quoi l'humain habite collectivement le monde, les «circuits de transindividuation ", comme dit Bernard Stiegler, œuvres d'art et objets techniques, institutions et réseaux de communication compris.

13. On consultera « why the future doesn't need us? » de Bill Joy (l'auteur du programme Java chez Sun Microsystems) Wired, Issue 8.04, April 2000, http://www.wired.com/wired/archive/ 8.04/joy.html ; voir aussi Allan Goldstein, "I nanobot ", et l'ouvrage de l'astronome du roi Sir Martin Rees, Our Final Century.

14. C'est-à-dire comme si le sujet humain pouvait disposer librement de n'importe quel objet technique comme d'un outil, et comme si la réalité humaine pouvait être modifiée directement et à volonté par une opération technique sur l'individu humain. On consultera A. Nordmann : « Ignorance at the heart of science? Incredible Narratives on Brain-machine Interfaces », 2006. http://www.uni-bielefeld.de/(en)/ZIF/FG/2006Application/PDF/Nordmann_essay.pdf

15. «FUTURISTIC THINKING is crucial to making the big leaps. It gives us some wild and crazy goals-a holy grail to chase. And the hope of glory propels us onward. Yet the famous 19thcentury chemist Kekulé once said, "Let us learn to dream, gentlemen, then perhaps we shall find the truth... But let us beware of publishing our dreams before they have been put to the proof by the waking understanding" ». Michael Roukes, "Plenty of room indeed », Scientific American, 2001, p. 42-49.

16. Éric K. Drexler, 1986, Engines of Creation, the Coming Era of Nanotechnology, traduction Engins de Création, l'avènement des nanotechnologies, Paris, Vuibert, 2005, p. 58.

17. Ibid., p. 57. L'idée de Drexler est que l'on ne peut pas prévoir les développements futurs des sciences. Il s'appuie sur Karl Popper : si on pouvait les prévoir, il n'y aurait nulle découverte en science ; en science, seule la prospective à court terme est possible; il s'agit de se concentrer sur les théories qui sont testables avec un appareillage disponible ou pouvant l'être à court terme. Par contre la prospective technologique est beaucoup plus sûre : il est possible d'anticiper sur la technologie parce qu'elle n'est que le développement, l'application des possibilités ouvertes et 
rendues concevables par les sciences fondamentales à une époque donnée. Cela aurait l'avantage d'intéresser les scientifiques aux avancées technologiques à long terme, dit Drexler. Si, aujourd'hui, les ingénieurs conçoivent en général des dispositifs à partir des possibilités d'application du présent, rien ne les empêche de concevoir des dispositifs à partir des possibilités d'application du futur, étant donné un cadre scientifique a peu près fixe. Les nanotechnologies deviennent alors une « science appliquée théorique » (theoretical applied science), parce qu'elles intègrent les concepts du futur (pour Drexler il s'agit de simulations informatiques basées sur des calculs dépendant des théories en vigueur) dans les réalisations du présent. Autrement dit, les réalisations du présent (comme les machines moléculaires qui existent) sont évaluées et jaugées relativement à un concept idéal d'application future.

18. Peu de recherches «nano» donnent lieu à des applications autres que des dispositifs... pour la recherche.

19. Gilbert Simondon, "Psychosociologie de la technicité ", Bulletin de l'École pratique de psychologie et de pédagogie de Lyon, $\mathrm{n}^{\circ}$ 2, novembre-décembre 1960, p. 127-140; n 3, janvier-février 1961, p. 227-238; nº 4, mars-juin 1961, p. 319-350.

20. Dans «Psycho-sociologie de la technicité », la culture avec un petit «c » désigne la culture incomplète ( «symbole de culture »). Définie par opposition à la technique réduite à un rôle «purement instrumental », la culture est «présentée comme source d'images et d'archétypes, tandis que les techniques définissent seulement la civilisation. En fait, culture et civilisation sont des symboles réciproques et complémentaires dont la réunion seule doit être considérée comme la culture au sens majeur du terme, c'est-à-dire au sens des anthropologues, des ethnologues, des ethnographes. La Culture, au sens majeur, comporte, comprend, et réunit la civilisation et la culture, au sens mineur du terme. » (Gilbert Simondon, «Psycho-sociologie de la technicité »).

21. On consultera pour cela l'article de Brigitte Nerlich, «From Nautilus to Nanobo(a)ts: The Visual Construction of Nanoscience ", http://www.azonano.com/details.asp?ArticleID=1466 en particulier la figure 7, qui montre un nanorobot (plutôt kitsch) choisissant un spermatozoïde pour le propulser vers l'ovule avant les autres, chevauchant littéralement l'Évolution!

22. C'est le cas de la nano-voiture de James Tour ou de la brouette moléculaire de Christian Joachim.

23. Si la chimie est une technologie, c'est aussi une grammaire qui dit les opérations de la matière. Il faut donc tenter d'expliquer ce dont il s'agit dans le langage des chimistes. La chimie de coordination, dont les concepts on presque un siècle, est concernée par les interactions entre molécules organiques (composés des éléments $\mathrm{C}, \mathrm{H}, \mathrm{O}$, et $\mathrm{N}$ ) et ions métalliques (atomes de métal chargés positivement ou négativement). Elle s'illustre par la synthèse de complexes de coordination, union d'un métal et de molécules organiques par des liaisons de coordination. Un complexe organo-minéral est composé d'un ion métal, dit de transition (cuivre, zinc, ruthénium), entouré de fragments organiques, les ligands (ou coordinats). Les ligands sont des fragments organiques porteurs de fonctions, c'est-à-dire de points disponibles pour d'autres liaisons moléculaires. Les ligands utilisés sont des chélates possédant au moins deux points de coordination, c'est-à-dire les points où ils se lient aux ions (ces points sont en général des atomes d'azote). Au LCOM ces ligands sont la bi-pyridine (2 points de coordination), la terpyridine (3) et la phénentroline (2). Par exemple, l'ion $\mathrm{Cu}(\mathrm{I})$ étant tétracoordiné, il se lie statistiquement avec 4 atomes d'azote, cela permet de faire des complexes phén-cuivre-phén ou des complexes phénecuivre-bipy. Dans l'exemple que nous décrivons (c'est l'exemple des figures 2 et 3), il s'agit de faire passer le métal de transition (le cuivre donc) du complexe phen-Cu(I)-bipy au complexe phen-Cu(II)-terpy, une fois qu'il est oxydé (il passe de I à II, c'est-à-dire perd un électron). C'est en jouant avec le nombre d'oxydation des ions et le nombre de points de coordination proposés à l'ion que l'on synthétise et fait fonctionner ces machines moléculaires. 
24. La nomenclature, par exemple $(1,10)$, indique la place des azotes sur chaque cycle, afin de distinguer les différents isomères. Par commodité, les atomes de $\mathrm{C}$ et de $\mathrm{H}$ ne sont pas représentés en chimie organique.

25. La chimie supra-moléculaire est la chimie des interactions non covalentes et des liaisons faibles en général - liaison hydrogène comme entre les bases de l'ADN, liaison van der Waals, certaines liaisons coulombiennes (ion/ion, ion/dipôle), et dipolaires (dipôle/dipôle), aromatiques ou basées sur l'hydrophobie. Celles-ci composent des objets, des architectures et des systèmes dits supra-moléculaires, liant plusieurs molécules en un assemblage facilement réversible. Elle a été inaugurée et popularisée par Jean-Marie Lehn (Nobel de Chimie 1987).

26. Le Microscope à effet tunnel (STM pour Scanning Tunneling Microscope) est l'instrument fétiche des nanotechnologies. Il fonctionne comme une sonde: une pointe terminée par un seul atome est approchée à quelques nanomètres d'une surface conductrice ou semi-conductrice, captant les électrons émis par effet tunnel, un champ électrique très faible à la surface des conducteurs; le «tunnel » est une métaphore pour désigner le fait que les électrons franchissent une barrière de potentiel. On approche la pointe jusqu'à établir un courant d'intensité défini entre la surface et la pointe, dit « courant de consigne ». On demande au mécanisme de contrôle (dont la partie active est constituée de tubes piézoélectriques, réagissant à une tension par une déformation mécanique et inversement) de conserver ce courant de consigne. Ce sont les déplacements en hauteur que la pointe a dû effectuer pour garder le courant constant qui sont enregistrés et traités pour reconstituer une topographie de la surface. On peut également balayer une surface en hauteur constante, mesurer les variations de courant tunnel et utiliser les interactions électroniques entre la pointe et les atomes pour attirer ou repousser les atomes ou les molécules qui y sont déposées.

27. Il est impossible d'avoir une «video » directe du mouvement d'une molécule : les dimensions des objets sont bien en deçà des longueurs d'onde de la lumière visible.

28. Cela peut être un taux très faible, comme $5 \%$ par exemple. Si une réaction à rendement faible intervient au début de la synthèse, il faut alors la reproduire, refaire la même manipulation autant de fois qu'il est nécessaire pour constituer un stock de molécules suffisant pour continuer à avancer en dans la synthèse avec l'espoir obtenir quelque chose à la fin.

29. C'est le cas par exemple de la DFT (Density-Functional Theory), théorie fonctionnelle de la densité, ou de la MBPT, (Many-Body Perturbation Theory), la théorie des perturbations pour un grand nombre de corps. Comme on ne sait pas résoudre l'équation de Shrödinger à $\mathrm{N}$-corps, ces théories sont en fait des manières de choisir les bonnes approximations, soit en partant des seules équations de la physique quantique dont on va simuler la résolution (méthodes ab initio), soit en modélisant la résolution des équations en se basant sur les expériences déjà réalisées (méthodes semi-empiriques).

30. Yoshida, M., Noji, H., Yasuda, R. Kinosita, K. JR.: 1997, « Direct observation of the rotation of F-ATPase ", Nature, 386 (1997) 299-302.

31. Ce qui peut être interprété dans deux sens pour un biologiste : soit les machines du vivant sont, comme le pense Jones, parfaitement optimisées (c'est le paradigme «panglossien» de l'évolution que critique Gould), soit elles sont des vestiges primitifs de l'évolution, hypertéliques, suradaptées et donc inévolutives, soumises à une " path-dependancy ». C'est ce que dit Drexler : la nature est un système technique conservateur, qui n'est pas le meilleur et n'est resté le même que parce que remplacer les anciennes machines, changer les moyens de production et former de nouveaux ouvriers représente un investissement trop lourd. La bonne vision est sûrement entre les deux, ni parfaitement optimisée, ni complètement archaïque, ces deux vues étant - quoique de manière différente - toutes deux anthropomorphiques.

32. p. 199-200, ce cours a été publié au Seuil dans la collection « traces écrites » en 2002.

33. «Partenaires » ou « associés » en Italien.

34. Pour le biphényl « relooké », voir : http://www2.cnrs.fr/presse/communique/674.htm 


\section{AUTEUR}

\section{SACHA LOEVE}

Doctorant en philosophie à Paris $\mathrm{X}$ 